\title{
Dahms, Regina (2019). Religiöse Vorstellungen von jungen Menschen mit vielseitig undefinierten Begabungen. Eine qualitative Untersuchung. Hildesheim: Olms Verlag. ISBN 978-3487 I 5793 I. 450 Seiten.
}

\section{Britta Konz}

Technische Universität Dortmund (Britta.Konz@tu-dortmund.de)

Regina Dahms widmet sich in ihrer qualitativen Studie der Frage, über welche religiösen Vorstellungen, welches Gotteskonzept junge Menschen mit vielseitig undefinierbaren Begabungen verfügen. Hierbei fokussiert sie auf den Berufsbildungsbereich der Werkstätten für Menschen mit Behinderung. Mit ihrer Studie leistet sie einen Beitrag zur Grundlagenforschung, gibt es bislang eher Studien über Menschen mit sogenannter "geistiger Behinderung", selten wird mit ihnen gesprochen und ihre Perspektive eingefangen. Unter dem Gotteskonzept versteht Dahms mit Astra Dannenfeldt und Bernhard Grom die in Wechselbeziehung stehende kognitive und emotional-motivationale Dimension des Glaubens, d. h., sie untersucht, wie Gott von den Befragten gedacht wird und welche Rolle er in ihrem Leben spielt.

Methodisch orientiert sich Dahms am qualitativ-heuristischen Forschungsansatz von Gerhard Kleining, den sie mit dem von Anna-Katharina Szagun entwickelten Rostocker Methodenensembles stützt. Da bei den Befragten (noch) keine Lese- und Schreibkompetenz vorausgesetzt werden konnte, wurde das Gespräch durch Visualisierungen mittels Materialcollagen gestützt. Ein weiterer Gesprächsstimuli waren Fotos, die zu den Impulsen „,welches Foto könnte Gott traurig/ froh machen?“ sortiert und zum Gesprächsanlass wurden. Damit sollten Möglichkeiten einer non verbalen Mitteilung von Gedanken und Gefühlen, der „bewussten und unbewussten gedanklichen Konstrukte“ (153) eröffnet werden. Ergänzend führte Dahms Elterngespräche, um Auskünfte über die religiöse Sozialisation sowie mögliche bedeutsame oder belastende Lebenssituationen zu erhalten. Für die Auswertung der qualitativen Interviews konnte Dahms auf jeweils fünf Transkriptionsprotokolle von Gesprächen mit 11 Personen zwischen 19 und 23 Jahre zurückgreifen, die im Laufe mehrerer Monate geführt wurden.

Das Buch führt in beeindruckender Weise vor Augen, wie vielschichtig theologische Gespräche mit jungen Menschen mit vielseitig undefinierten Begabungen sein können. Ein Fundus für das religionspädagogische Arbeiten sind die Einzelfallstudien, in denen Dahms Interviewsequenzen auswertet und Fotos der erstellten Materialcollagen und der Positionierungsübung präsentiert. Sie bestätigen die Forschung Stefan Anderssohns, dass neben der kognitiven Struktur die Lebens-Themen bedeutsam sind für die Herausbildung von Gottesvorstellungen, weshalb Schulmaterial sich nicht einfach an dem von Grundschüler*innen orientieren darf. In den Interviews der qualitativen Studie waren es Themen wie Liebe, Freundschaftsbeziehungen, Streit, Ausgrenzung, Trennung und Tod, die von den Interviewten in die theologischen Gespräche eingebracht wurden. So beschreibt beispielsweise Andrea ihre ambivalente Reaktion auf den Tod des Familienhundes, den sie traurig fand, aber auch als Erlösung für den Hund deutete. Bedeutsam war für sie, dass sie sich von ihm auf eine gute Weise verabschieden konnte. Gott kommuniziert ihrer Ansicht nach mit ihr und anderen Menschen durch ein Kribbeln oder einen kleinen Stich ins Herz. Erik räumt ein, dass Gott nicht da war, als er Angst vor einem Mitbewohner hatte, wie auch in wütenden Situationen, wenn er sich von niemandem verstanden fühlt. Er glaubt jedoch, dass Gott ihm einen Engel geschickt hat: seine Freundin. Die Theodizeefrage wird als Verantwortung der Menschen gedeutet, auch ganz konkret, wenn z. B. Claudia auf die Frage, wer das Schlimme in der Welt bewirke, „Mein Ex-Freund“ antwortet (196). Gott wird von allen mit dem Guten assoziiert, der, wie einige betonen, dabei Hilfe von Polizei oder Bundeswehrsoldaten erhält. 
In den Einzelfallstudien wird sichtbar, dass auch Menschen mit vielseitig undefinierten Begabungen mit der Breite der Themen konfrontiert werden, die ihre Lebenswelt prägen und diese für sich verarbeiten. So finden sich in den Interviews beispielsweise Auseinandersetzungen mit Themen wie Terrorismus. Gott wünsche sich, argumentiert Peter, dass die Menschen friedlich, ohne Krieg und Terror miteinander leben, er mutmaßt aber, dass es wohl immer irgendwo Konflikte geben werde (321). Dabei führt er an, dass "die ISIS“ ja „keine richtige Religion“ sei (321). Möglicherweise bezieht er sich hierbei auf die Kritik an einer Gleichsetzung der Terrororganisation Islamischer Staat mit dem Islam.

Dahms schlussfolgert für die religionspädagogische Arbeit, dass biblische Themen wiederholt eingebracht werden müssten, weil Inhalte teilweise unvollständig oder fehlerhaft zusammengefügt und wiedergegeben werden. So mutmaßt z. B. Claudia, dass die Hirten gefährlich seien und das Baby Jesus getötet haben. Möglicherweise referenziert sie dabei auf den Kindermord in Bethlehem (Mt 2, 13-18), wie Dahms vermutet, oder fügt Fragmente der Weihnachtsgeschichte und Ostererzählung zusammen. Des Weiteren empfiehlt Dahms die Förderung ethischer Reflexionen, das Gestalten inklusiver Gottesdienste und Konfirmandenunterrichte und vor allem eine Begleitung bei Fragen zu Sterben und Tod. Alle bis auf einer der elf Gesprächspartner*innen hatten schon Erfahrungen mit dem Tod von Eltern oder nahen Angehörigen gemacht und sind auf der Suche nach Antworten. Dadurch, dass Dahms über einen Zeitraum von mehreren Monaten Gespräche führte, ließen sich auch Weiterentwicklungen der Gotteskonzepte durch die wiederholten Gespräche feststellen, was die Bedeutung religiöser Bildungsangebote bestätigt.

Das Buch bietet einen umfassenden Einblick in den neuesten Stand der Forschung zu religiösen Vorstellungen von Menschen mit vielseitig undefinierten Begabungen und leistet einen Beitrag dazu, die Forschungslücke qualitativer Befragungen von Menschen mit "geistiger Behinderung“ zu füllen und das methodische Instrumentarium hierfür weiterzuentwickeln. Aufgrund des umfangreichen empirischen Materials, das in seiner Tiefe noch gar nicht vollends ausgeschöpft ist, lädt das Buch auch über die berufsschulische Begleitung hinaus dazu ein, inklusive empirische Forschungsformate und Didaktiken weiterzuentwickeln. 